\title{
Scattered compactification for the Arens' space $S_{2}$
}

by

M. Jay achandran and M. Rajagopalan* (Memphis, Tenn.)

Abstract. We prove, using $(\mathrm{CH})$, that the scattered Hausdorff space $S_{2}$, introduced by R. Arens n 1950, admits a scattered Hausdorff compactification. This gives an affirmative answer to a problem aised by Z. Semadeni in 1959.

Introduction. The class of scattered spaces has been recently studied by several mathematicians like V. Kannan [2], S. Mrówka, M. Rajagopalan, T. Soundararajan ([3], [5]), C. Ryll-Nardzewski, R. Telgarsky [6], Z. Semadeni [7] and W. Sierpiński [8]. It has been recently proved by P. J. Nyikos [4] and R. C. Solomon [9] that there exists a completely regular scattered space which does not admit a scattered compactification. However, the problem of deciding whether or not a given completely regular scattered space admits a scattered Hausdorff compactification is a nontrivial one. In this connection, S. P. Franklin has raised the following question: "Does the Aren's space $S_{2}$ (definition follows) admit a Hausdorff scattered compactification?" Z. Semadeni [7] has raised the question whether a particular subspace of $S_{2}$ has a scattered compactification. The aim of this paper is to give an affirmative answer to these questions, by constructing a suitable quotient space $X$. of $\beta N$, the Stone-Čech compactification of the set $N$ of natural numbers, such that $X$ is scattered and $X$ contains a homeomorph of $S_{2}$ as a dense subspace.

Notation 1. $N$ denotes the discrete space of natural numbers and $\beta N$, its StoneCech compactification. $\omega$ denotes the least infinite ordinal and $\Omega$ denotes the first uncountable ordinal. For any subset $A \subseteq N, A^{*}$ denotes the set

$$
\left(\mathrm{cl}_{\beta N} A\right) \cap(\beta N-N)=\left(\mathrm{cl}_{\beta N} A\right)-A \text {. }
$$

DefinItIon 2. We denote by $S_{1}=N \cup\{\infty\}$, the one-point compactification of $N . S_{1}$ is also called a convergent sequence and the only non-isolated point $\infty$ of $S_{1}$ is called its suspension point.

Defrnrtion 3, For each $n \in N$, let $X_{n}$ denote a homeomorphic copy of $S_{1}$. Let $X_{n} \cap X_{m}=\varnothing$ for all $n, m \in N$ such that $n \neq m$. Also, let $x_{n} \in X_{n}$ be the suspen-

* The second author acknowledges gratefully a grant from Memphis State University during the preparation of this paper.

5 - Fundamenta Mathematicae XCVI 
sion point of $X_{n}$ for each $n \in N$, Let $C=\left\{c_{1}, c_{2}, \ldots, c_{n}, \ldots\right\} \cup\left\{c_{\infty}\right\}$ be a convergent sequence such that $c_{\infty}$ is the suspension point of $C$ and $C \cap X_{n}=\varnothing$ for all $n \in N$. Let $B$ denote the free union $\left(\bigcup_{n=1}^{\infty} X_{n}\right) \cup C$ of the spaces $X_{n}$ with $C$. Define a partition $\pi$ in $B$ by declaring $\left\{c_{n}, x_{n}\right\}$ as a member of $\pi$ for each $n \in N$ and $\{x\}$ as a member of $\pi$ for all other $x \in B$. The quotient space $B / \pi$ is called the Arens' space $S_{2}$ (see [1]).

Construction $4(\mathrm{CH})$. Take a sequence $A_{1}, A_{2}, \ldots, A_{n}, \ldots$ of mutually disjoint countably infinite subsets of $N$ whose union is $N$. Then each $A_{n}^{*}$ is clopen in $\beta N-N$ and $A_{n}^{*} \cap A_{m}^{*}=\varnothing$ for $n \neq m, n, m=1,2, \ldots$ Let $M=\operatorname{cl}_{\beta N}\left(\bigcup_{n=1}^{\infty} A_{n}^{*}\right)$ and $K=M-\bigcup_{n=1}^{\infty} A_{n}^{*}$. Then $K$ is a non-empty closed set in $\beta N-N$ and it is not open in $\beta N-N$. Let $\mathscr{F}=\left\{S \subseteq N / S\right.$ is infinite and $S \cap A_{n}$ is finite $\left.\forall n=1,2, \ldots\right\}$. Then, $\forall S \in \mathscr{F}, S^{*}$ is clopen in $\beta N-N$ and $S^{*} \cap A_{n}^{*}=\varnothing$ for all $n=1,2, \ldots$ Now, well order $\mathscr{F}$. Let us assume continuum hypothesis. Then $\mathscr{F}$ can be written as $\mathscr{F}=\left\{S_{\alpha} \mid \alpha \in[1, \Omega)\right\}$. Put $T_{\alpha}=S_{\alpha}^{*}$ for all $\alpha \in[1, \Omega)$. For $\alpha \in[1, \Omega)$, define the sets $F_{\alpha} \subset \beta N-N$ as follows: Put $F_{1}=T_{1}$. Having chosen $F_{i}$ for each $i, 1 \leqslant i<\gamma, \gamma \in[1, \Omega)$, choose $F_{\gamma}$ such that $F_{\gamma}$ is clopen in $\beta N-N, F_{\gamma} \cap M=\varnothing$, and $F_{\gamma} \supseteq \bigcup_{1 \leqslant i<\gamma} F_{i} \cup T_{\gamma}$. This is possible, since the Boolean algebra of clopen sets in $\beta N-N$ is Dubois-Reymond separable [10]. Our construction ends here.

THEOREM 5. The space $S_{2}$ admits a scattered Hausdorff compactification.

Proof. Let the sets $S_{\alpha}, T_{\alpha}, F_{\alpha}(\alpha \in[1, \Omega)), M, K$ and $A_{n}$ be as constructed above. Then,

Clatm 1. $\underset{\alpha \in[1, \Omega)}{\bigcup} F_{\alpha}=\beta N-N-M$. For, it is clear that $\bigcup_{\alpha \in[1, \Omega)} F_{\alpha} \subset \beta N-N-M$. To get the other inclusion, let $x_{0} \in \beta N-N-M$. Now, $\beta N-N$ is zero dimensional, $M$ is closed in $\beta N-N$ and $x_{0} \notin M$. Therefore, there exists a clopen set $V$ in $\beta N-N$ such that $x_{0} \in V$ and $V \cap M=\varnothing$. Also, $V=A^{*}$ for some infinite subset $A$ of $N$ and $V \cap A_{n}^{*}=\varnothing$ for all $n=1,2, \ldots$ Hence, $A \cap A_{n}$ is finite for all $n=1,2, \ldots$ So, $A=S_{\alpha}$ for some $\alpha \in[1, \Omega)$ and hence, $V=T_{\alpha} \subseteq F_{\alpha}$. This implies $x_{0} \in F_{\alpha}$ and our claim is justified.

Clarm 2. There exists a compact, Hausdorff space $X$ and a map $q: \beta N \rightarrow X$ such that

(i) $q$ is a quotient map and

(ii) $q(\beta N-N)$ is homeomorphic to the quotient space obtained by taking the free union of $[1, \omega]$ and $[1, \Omega]$ with their usual order topologies and identifying $\omega$ and $\Omega$.

To justify this claim, we can assume, without loss of generality, that the sets $F_{\alpha}$ of our construction are all distinct. Now, put $H_{1}=F_{1}$ and $H_{\alpha}=F_{\alpha}-\bigcup_{1 \leqslant i<\alpha} F_{i}$ for all $\alpha$ such that $2 \leqslant a<\Omega$. Then $\underset{\alpha \in[1, \Omega)}{\bigcup} F_{\alpha}=\bigcup_{\alpha \in[1, \Omega)} H_{\alpha}$. Further, the disjoint collection of sets $\left\{H_{\alpha}\right\}_{\alpha \in[1, \Omega),},\left\{A_{n}^{*}\right\}_{n \geqslant 1},\{K\}^{\alpha \in[1, \Omega)}$ and $\{n\}_{n \in N}, n \in N$ gives a partition of $\beta N$ by closed sets in $\beta N$. Let the quotient space induced by this partition be denoted by $X$. Let $q: \beta N \rightarrow X$ be the corresponding quotient map. Then, it is clear that $X$ is compact and Hausdorff. Let $q\left(A_{n}^{*}\right)=\left\{l_{n}\right\}$ for all $n \in N ; q(K)=\left\{l_{\infty}\right\} ; q\left(H_{\alpha}\right)=\left\{l_{\alpha}\right\}$ for all $\alpha \in[1, \Omega)$. Then, we have $q(\beta N-N)=\left\{I_{1}, l_{2}, \ldots, I_{n}, \ldots\right\} \cup\left\{I_{\infty}\right\} \cup\left\{I_{\alpha}\right\}_{1 \leqslant \alpha<\Omega}$ and it can be verified that $q$ satisfies the conditions (i) and (ii) of Claim 2 .

ClaIm 3. If $q(M \cup N)=Y$, then $Y$ is homeomorphic to $S_{2}$. To justify this claim, let us take $A_{k}=\left\{a_{k_{1}}, a_{k_{2}}, \ldots, a_{k_{n}}, \ldots\right\}$ for all $k=1,2, \ldots ; q\left(A_{n}\right)=B_{n}$ for all $n \dot{E} N$ and $q\left(a_{k_{n}}\right)=l_{k_{n}}$ for all $k, n \in N$. Then, each $\left\{l_{m_{n}}\right\}, m, n \in N$, is clopen in $Y$. Also, we note that $l_{n} \rightarrow l_{\infty}$ as $n \rightarrow \infty$. Further, the compactness of $q\left(A_{n} \cup A_{n}^{*}\right)$ implies that $l_{n}=\lim _{k \rightarrow \infty} l_{n_{k}}$ for all $n=1,2, \ldots$

We will now show that an open set in $Y$ containing $l_{\infty}$ is an open set containing $l_{\infty}$ in the topology of $S_{2}$ and vice versa. Let 0 be an open set in $Y$ such that $l_{\infty} \in 0$. Since, $l_{n} \rightarrow l_{\infty}$ as $n \rightarrow \infty$, there exist $n_{0} \in N$, such that $l_{n} \in 0$ for all $n \geqslant n_{0}$. Since $l_{n_{k}} \rightarrow l_{n}$ as $k \rightarrow \infty$, for all $n \in N$, it follows that $(Y-0) \cap B_{n}$ is finite for all $n \geqslant n_{0}$. Conversely, let $S \subseteq q(N)$ be such that $S \cap B_{n}$ is finite for all $n \in N$. Then $q^{-1}(S) \cap A_{n}$ is finite for all $n \in N$. Therefore, $q^{-1}(S)=S_{\alpha}$ for some $\alpha \in[1, \Omega)$. Also $S_{\alpha}^{*}=T_{\alpha} \subseteq F_{\alpha}$ and so

$$
\mathrm{cl}_{\beta N}\left(S_{\alpha} \cup F_{\alpha}\right)=\mathrm{cl}_{\beta N}\left(S_{\alpha}\right) \cup F_{\alpha}=S_{\alpha} \cup S_{\alpha}^{*} \cup F_{\alpha}=S_{\alpha} \cup F_{\alpha} .
$$

Therefore, $S_{\alpha} \cup F_{\alpha}$ is closed in $\beta N$ and hence $q\left(S_{\alpha} \cup F_{\alpha}\right)$ is closed in $X$. Therefore, $\left(S \cup q\left(F_{\alpha}\right)\right) \cap Y$ is closed relative to $Y$. But $F_{\alpha} \cap M=\varnothing$ for all $\alpha \in[1, \Omega)$ implies that $q\left(F_{\alpha}\right) \cap Y=\varnothing$. Therefore, $S \cap Y=S$ is closed relative to $Y$. Hence, $Y-S$ is open in $Y$ and $l_{\infty} \in Y-S$. Therefore, the relative topology of $Y$ from $X$ is homeomorphic to $S_{2}$.

Since $N$ is dense in $\beta N$, we also have $q(M \cup N)=Y$ is dense in $X$. Since, the relati ology of $q(N)$ from $X$ is discrete and that of $q(\beta N-N)$ is homeomorphic to th : obtained by taking the free union of $[1, \omega]$ and $[1, \Omega]$ and identifying $\omega$ and $\quad \operatorname{im} 2$ ), it follows that $X$ is scattered. This completes the proof of the the

\section{References}

.. Arens, Note on convergence in topology, Math. Mag. 23 (1950), pp. 229-234. V. Kannan and M. Rajagopalan, On scattered spaces, Proc. Amer. Math. Soc. 43 (1974), pp. $402-408$.

S. Mrówka, M. Rajagopalan and T. Soundararajan, A characterization of compact scattered spaces through chain limits, Topology 72, Springer-Verlag, Lecture Notes in Mathematics, Vol. 378 (1974), pp. 288-298.

r] P. J. Nyikos, Not every scattered space has a scattered compactification, Notices of Amer. Math. Soc. 21 (1974), p. 570.

[5] M. Rajagopalan, Sequential order and spaces $S_{n}$, Proc. Amer. Math. Soc. 54 (1976), pp. 433-438.

[6] C. Ryll-Nardzewski and R. Telgarsky, On scattered compactification, Bull. Acad. Polon. Sci. Sér. Sci. Math. Astronom. Phys. 18 (1970), pp. 233-234. 
[7] Z. Semadeni, Sur les ensembles clairsemés, Dissertationes Math. 19 (1959), p. 39

[8] W. Sierpiński and S. Mazurkiewicz, Contribution à la topologie des ensembles dénombrables, Fund. Math. 1 (1920), pp. 11-16.

[9] R. C. Solomon, Scattered spaces and their compactifications, to appear.

[10] R. C. Walker, The Stone-Čech compactification, (Thesis) Department of Mathematics, Carnegie-Mellon University, (1972).

\section{MADURA COLLEGE}

\section{The equivalence of absolute almost continuous retracts and $\varepsilon$-absolute retracts}

by

Kenneth R. Kellum* (Birmingham, Ala.)

Abstract. In this paper we are concerned with types of generalized retracts and $\varepsilon$-retracts which the in which the res ef functions which is assumed of retract in which the retraction function belongs to an arbitrary class of functions which is assumed to be closed under composition with continuous functions. Theorems are proved which are generalizations of well-known theorems about AR's and $\varepsilon$ AR's. These theorems hold if the class in question is the class of the conther proposition which is our title, lead to a number of other equivalences.

1. Introduction. In [11] I reported that an almost continuous retract of an $n$-cube need not be compact. These spaces are of interest because they must posses the fixed point property. The present paper is the result of studying the special case of those almost continuous retracts which do happen to be compact. The main result implies that a compact subset $Y$ of an $n$-cube $X$ is an almost continuous retract of $X$ if and only if $Y$ is an $\varepsilon$-retract of $X$.

Suppose $Y \subset X$. That $Y$ is a retract of $X$ means that there exists a continuous function (called a retraction) $r: X \rightarrow Y$ such that $x=r(x)$ for each $x \in Y$. This important concept is due to Borsuk and has been studied extensively (see [1] and [9]). Recently the notion of a retract has been generalized in two seemingly different ways. First, Noguchi [16] and later Gmurczyk [4], [5] and Granas [6] studied $\varepsilon$ - (or approximative) retracts in which the requirement that $x=r(x)$ is weakened. Second, motivated by question 10 of Stallings [17], several authors have studied connectivity and almost continuous retracts in which the requirement that the retraction function be continuous is weakened (see [2], [3], [7], [10], [11] and [12]). Here we show that these two lines of research are in fact closely related.

We adopt the following conventions. All spaces, except the function spaces considered below, are assumed to be separable metric. If $x$ and $y$ are points of a space $X, d(x, y)$ denotes the distance from $x$ to $y$. If $x \in X$, then

$$
N(x, \varepsilon)=\{y \in X: d(x, y)<\varepsilon\} .
$$

* Author supported in part" by NSF Grant GY-10729. 